\title{
Medium Effects on the Decarboxylation of a Biotin Model in Pure and Mixed Solvents from QM/MM Simulations
}

\author{
Orlando Acevedo and William L. Jorgensen* \\ Department of Chemistry, Yale University, 225 Prospect Street, \\ New Haven, Connecticut 06520-8107.
}

Supporting Material

Figure S1. Solute-solvent energy pair distribution in methanol............................ 1

Figure S2. Solute-solvent energy pair distribution $33 \%$ and $67 \%$ methanol........................S2

Figure S3. Solute-solvent energy pair distribution $33 \%$ and $67 \%$ acetonitrile.......................S3

Cartesian coordinates for the biotin-model reactant with 8 water molecules (Figure 4).........S4

Cartesian coordinates for the biotin-model TS with 8 water molecules (Figure 4)................S5

Cartesian coordinates for the biotin-model TS in 67\% aqueous acetonitrile (Figure 8)..........S6



Figure S1. Solute-solvent energy pair distributions for the decarboxylation of $\mathbf{2}$ in methanol for the reactant, transition structure (TS), and products. The ordinate records the number of solvent molecules that interact with the solutes with their interaction energy on the abscissa. Units for the ordinate are number of molecules per $\mathrm{kcal} / \mathrm{mol}$. 

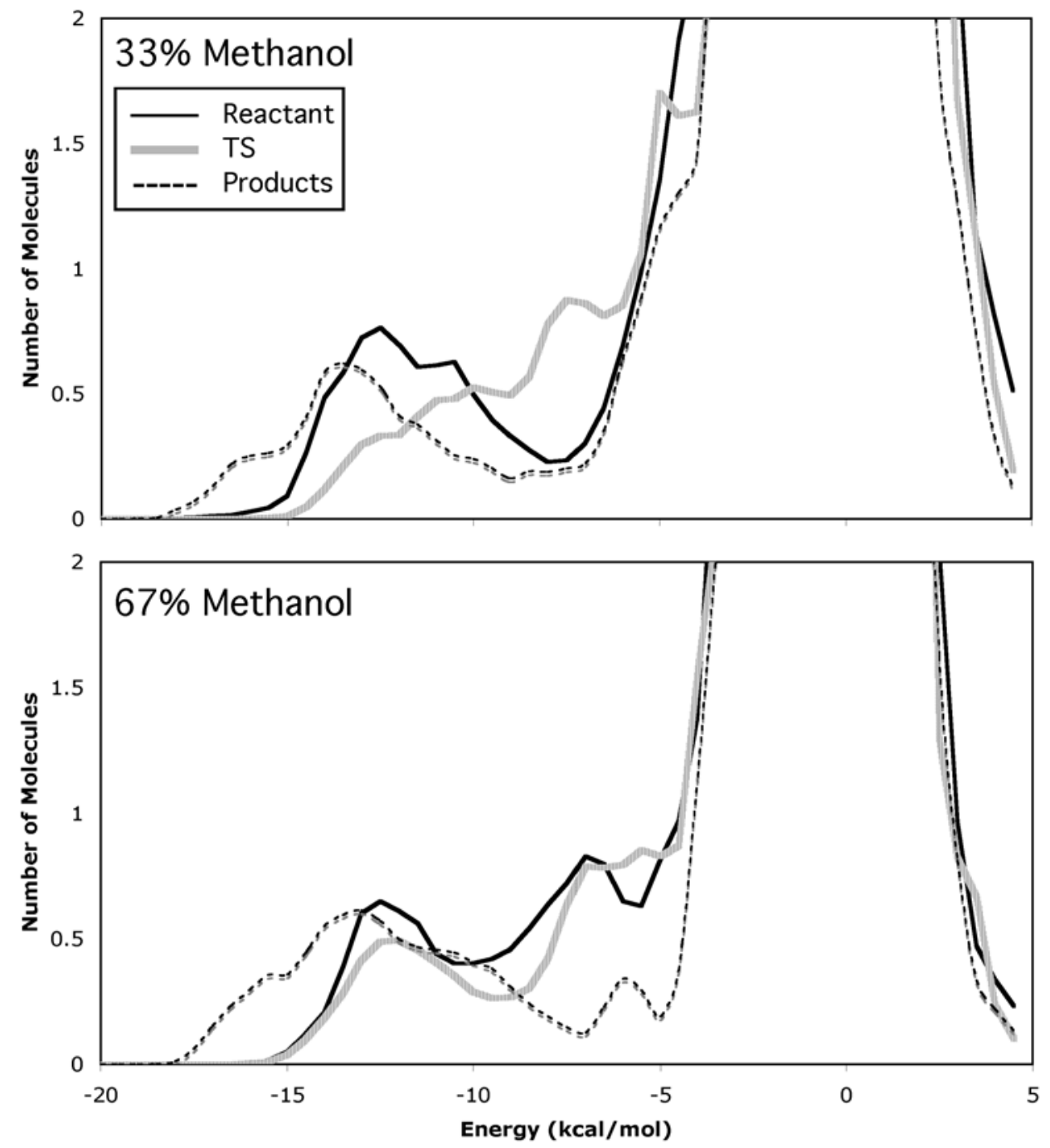

Figure S2. Solute-solvent energy pair distributions for the decarboxylation of $\mathbf{2}$ in a 33\% and 67\% methanol to water mixture for the reactant, transition structure (TS), and products. The ordinate records the number of solvent molecules that interact with the solutes with their interaction energy on the abscissa. Units for the ordinate are number of molecules per $\mathrm{kcal} / \mathrm{mol}$. 

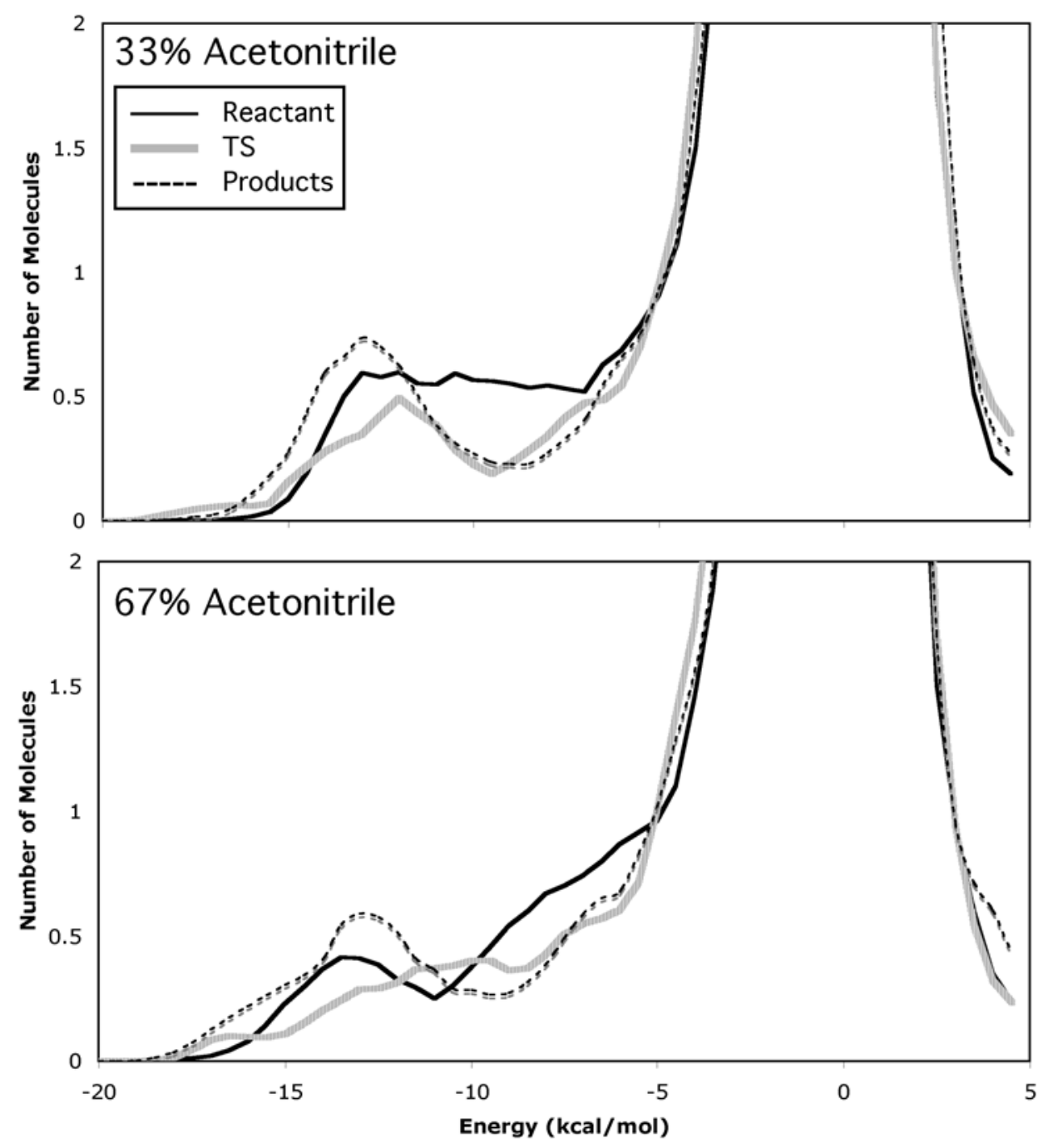

Figure S3. Solute-solvent energy pair distributions for the decarboxylation of $\mathbf{2}$ in a 33\% and 67\% acetonitrile to water mixture for the reactant, transition structure (TS), and products. The ordinate records the number of solvent molecules that interact with the solutes with their interaction energy on the abscissa. Units for the ordinate are number of molecules per $\mathrm{kcal} / \mathrm{mol}$. 


\section{Cartesian coordinates for the biotin-model reactant with 8 water molecules from Figure 4.}

38

C $\quad 0.952 \quad 1.131-0.385$

$\begin{array}{llll}N & -0.004 & 0.358 & -1.074\end{array}$

$\begin{array}{llll}N & 2.111 & 0.324 & -0.121\end{array}$

C $\quad 0.510-1.051-1.325$

$\begin{array}{llll}C & 1.933 & -1.082 & -0.763\end{array}$

$\begin{array}{llll}0 & 0.908 & 2.357 & -0.101\end{array}$

$\begin{array}{llll}C & -1.421 & 0.583 & -1.281\end{array}$

$\begin{array}{llll}0 & -1.943 & 1.697 & -0.911\end{array}$

$\begin{array}{llll}0 & -2.094 & -0.422 & -1.681\end{array}$

$\begin{array}{llll}\mathrm{H} & 2.977 & 0.790 & -0.269\end{array}$

$\begin{array}{llll}H & 2.756 & -1.135 & -1.537\end{array}$

$\begin{array}{llll}H & 2.065 & -1.864 & 0.068\end{array}$

$\begin{array}{llll}H & 0.468 & -1.189 & -2.442\end{array}$

$\begin{array}{llll}H & -0.126 & -1.822 & -0.903\end{array}$

$\begin{array}{llll}0 & -1.335 & -1.968 & -3.689\end{array}$

$\begin{array}{llll}H & -1.976 & -2.627 & -3.961\end{array}$

$\begin{array}{llll}H & -1.752 & -1.521 & -2.952\end{array}$

$\begin{array}{llll}0 & -3.323 & -1.004 & 0.593\end{array}$

$\begin{array}{llll}H & -2.897 & -0.735 & -0.221\end{array}$

$\begin{array}{llll}\mathrm{H} & -2.635 & -0.945 & 1.253\end{array}$

$\begin{array}{llll}0 & -4.690 & 1.271 & -1.360\end{array}$

$\begin{array}{llll}H & -5.296 & 1.872 & -0.925\end{array}$

$\begin{array}{llll}\mathrm{H} & -3.824 & 1.648 & -1.203\end{array}$

$\begin{array}{llll}0 & 4.654 & 0.667 & -1.357\end{array}$

$\begin{array}{llll}\mathrm{H} & 4.472 & 0.359 & -2.245\end{array}$

$\begin{array}{llll}\text { H } & 5.240 & 1.415 & -1.477\end{array}$

$\begin{array}{llll}0 & 1.385 & 4.274 & 2.294\end{array}$

$\begin{array}{llll}H & 1.052 & 5.136 & 2.047\end{array}$

$\begin{array}{llll}\text { H } & 0.956 & 3.668 & 1.688\end{array}$

$\begin{array}{llll}0 & 0.771 & 3.728 & -2.474\end{array}$

$\begin{array}{llll}\text { H } & 1.581 & 3.573 & -2.961\end{array}$

$\begin{array}{llll}\mathrm{H} & 0.820 & 3.125 & -1.733\end{array}$

$\begin{array}{lrrr}0 & -1.763 & 3.340 & 1.140\end{array}$

$\begin{array}{llll}\mathrm{H} & -1.812 & 2.722 & 0.411\end{array}$

$\begin{array}{llll}\mathrm{H} & -1.605 & 2.790 & 1.907\end{array}$

$\begin{array}{llll}0 & -3.041 & 3.655 & -2.277\end{array}$

$\begin{array}{llll}\mathrm{H} & -2.633 & 4.355 & -1.764\end{array}$

$\begin{array}{llll}H & -2.625 & 2.853 & -1.961\end{array}$ 
Cartesian coordinates for the biotin-model transition state with 8 water molecules from Figure 4.

38

$\begin{array}{rrrr}\mathrm{C} & -0.146 & -0.652 & 0.869 \\ \mathrm{~N} & -1.365 & -1.263 & 0.851 \\ \mathrm{~N} & 0.957 & -1.581 & 0.792 \\ \mathrm{C} & -1.102 & -2.751 & 0.524 \\ \mathrm{C} & 0.382 & -2.857 & 0.211 \\ 0 & 0.126 & 0.559 & 1.089 \\ \mathrm{C} & -3.092 & -0.173 & 0.245 \\ \mathrm{O} & -2.682 & 0.567 & -0.578 \\ \mathrm{O} & -3.865 & -0.755 & 0.944 \\ \mathrm{H} & 1.764 & -1.258 & 0.321 \\ \mathrm{H} & 0.672 & -2.925 & -0.877 \\ \mathrm{H} & 0.969 & -3.665 & 0.703 \\ \mathrm{H} & -1.743 & -3.152 & -0.275 \\ \mathrm{H} & -1.382 & -3.424 & 1.342 \\ \mathrm{O} & -2.899 & 1.275 & -3.355 \\ \mathrm{H} & -2.917 & 0.501 & -2.793 \\ \mathrm{H} & -2.367 & 1.013 & -4.107 \\ \mathrm{O} & 2.291 & 2.063 & 1.766 \\ \mathrm{H} & 1.735 & 2.666 & 2.261 \\ \mathrm{H} & 1.676 & 1.481 & 1.321 \\ \mathrm{O} & -3.783 & -0.181 & 3.870 \\ \mathrm{H} & -2.964 & -0.672 & 3.928 \\ \mathrm{H} & -4.032 & -0.243 & 2.947 \\ \mathrm{O} & -0.279 & 0.745 & 3.850 \\ \mathrm{H} & 0.003 & 0.326 & 3.038 \\ \mathrm{H} & 0.276 & 1.521 & 3.924 \\ \mathrm{O} & -1.835 & -2.209 & 3.390 \\ \mathrm{H} & -1.739 & -1.849 & 2.507 \\ \mathrm{H} & -0.956 & -2.501 & 3.624 \\ \mathrm{O} & 3.785 & -1.320 & -0.063 \\ \mathrm{H} & 3.517 & -2.211 & -0.284 \\ \mathrm{H} & 4.024 & -0.925 & -0.901 \\ \mathrm{O} & -0.509 & 2.135 & -0.927 \\ \mathrm{H} & -0.140 & 1.543 & -0.268 \\ \mathrm{H} & -1.290 & 1.683 & -1.243 \\ 0 & -0.900 & -1.097 & -2.241 \\ \mathrm{H} & -1.037 & -2.019 & -2.453 \\ \mathrm{H} & -1.093 & -1.032 & -1.307\end{array}$


Cartesian coordinates for the biotin-model transition state in $67 \%$ aqueous acetonitrile from Figure 8.

$\begin{array}{rrrr} & & & \\ \mathrm{S3} & & & \\ \mathrm{C} & -0.453 & -0.181 & -0.648 \\ \mathrm{~N} & 0.660 & -0.894 & -1.069 \\ \mathrm{~N} & -1.706 & -0.939 & -0.886 \\ \mathrm{C} & 0.244 & -2.165 & -1.665 \\ \mathrm{C} & -1.345 & -2.250 & -1.443 \\ \mathrm{O} & -0.463 & 0.973 & -0.246 \\ \mathrm{C} & 2.708 & -0.311 & -1.010 \\ \mathrm{O} & 2.504 & 0.899 & -0.923 \\ \mathrm{O} & 3.223 & -1.382 & -1.083 \\ \mathrm{H} & -2.341 & -1.036 & -0.110 \\ \mathrm{H} & -1.641 & -3.156 & -0.795 \\ \mathrm{H} & -1.797 & -2.377 & -2.383 \\ \mathrm{H} & 0.855 & -2.882 & -1.110 \\ \mathrm{H} & 0.430 & -2.237 & -2.667 \\ \mathrm{O} & 1.431 & -1.729 & 1.631 \\ \mathrm{H} & 1.899 & -2.551 & 1.487 \\ \mathrm{H} & 1.198 & -1.426 & 0.754 \\ \mathrm{C} & 2.549 & -4.884 & -0.210 \\ \mathrm{~N} & 2.121 & -4.753 & 0.856 \\ \mathrm{C} & 3.086 & -5.050 & -1.556 \\ \mathrm{C} & 4.162 & 4.502 & -1.342 \\ \mathrm{~N} & 4.476 & 4.073 & -2.368 \\ \mathrm{C} & 3.766 & 5.047 & -0.049 \\ \mathrm{O} & 0.978 & 2.691 & 0.963 \\ \mathrm{H} & 0.396 & 2.016 & 0.612 \\ \mathrm{H} & 1.432 & 3.041 & 0.197 \\ \mathrm{O} & -2.801 & 1.820 & 0.788 \\ \mathrm{H} & -1.979 & 1.513 & 0.405 \\ \mathrm{H} & -3.368 & 1.986 & 0.035 \\ \mathrm{O} & -2.901 & 0.593 & -2.976 \\ \mathrm{H} & -0.034 & -0.106 & -3.222 \\ \mathrm{H} & -3.113 & 1.454 & -2.612 \\ \mathrm{H} & -2.329 & 0.192 & -2.322 \\ \mathrm{C} & 5.945 & 1.066 & -4.654 \\ \mathrm{~N} & 7.013 & 0.944 & -5.082 \\ \mathrm{C} & 4.597 & 1.220 & -4.117 \\ \mathrm{C} & -3.595 & -4.907 & -0.347 \\ \mathrm{~N} & -3.704 & -4.831 & -1.495 \\ \mathrm{C} & -3.458 & -5.001 & 1.102 \\ \mathrm{C} & 6.869 & 1.452 & -0.003 \\ \mathrm{~N} & 6.706 & 1.441 & 1.144 \\ \mathrm{C} & 7.073 & 1.465 & -1.446 \\ \mathrm{C} & -0.003 & -6.273 & -3.220 \\ \mathrm{~N} & 0.310 & -5.221 & -3.591 \\ \mathrm{C} & -0.396 & -7.596 & -2.753 \\ \mathrm{O} & -3.582 & -1.150 & 1.412 \\ -3.643 & -0.196 & 1.463 \\ \mathrm{H} & -4.215 & -1.395 & 0.738 \\ \mathrm{H} & -1.213 & 0.104 & -4.118 \\ \mathrm{H} & & -3.067\end{array}$


$\begin{array}{llll}\mathrm{N} & 1.727 & 3.392 & -3.763\end{array}$

$\begin{array}{llll}\text { C } & -0.358 & 3.504 & -2.190\end{array}$ 